\title{
Akustische Reize gegen den Radau im Ohr
}

Seit Kurzem ist eine neue Behandlungsmöglichkeit für Tinnitus auf dem Markt. Die Zwischenbilanz einer ersten klinischen Studie mit 45 Patienten scheint vielversprechend: Nach zwölf Wochen Therapie mit dem Neurostimulator hatten die Ohrgeräusche der Teilnehmer um 40 bzw. 33\% abgenommen, in der Placebogruppe dagegen nur um 9 bzw. 8\%. Zudem wurde die Tinnitusfrequenz zunehmend tiefer. Einige Patienten, bei denen das Ohrgeräusch über viele Jahre bestand, sind sogar vollständig beschwerdefrei.

Der Stimulator wird zur Behandlung von chronischem, subjektivem, tonalem Tinnitus eingesetzt. Dieser entsteht durch eine Fehlsteuerung krankhaft überaktiver Nervenzellen, die plötzlich hoch-

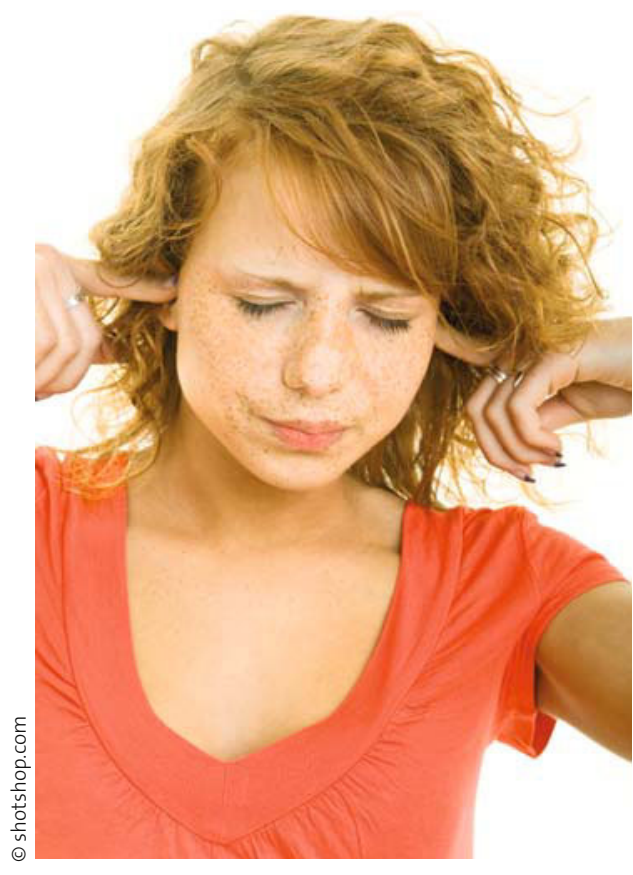

Das regelmäßige Hören einer leisen Tonfolge soll das Ohrgeräusch beseitigen. synchron arbeiten. Durch gezielte akustische Reize soll das "gesunde Chaos" in den Nervennetzwerken des Gehirns wiederhergestellt werden. Hierzu programmiert der HNO-Arzt den streichholzschachtelgroßen Neurostimulator mit einer Folge von Tönen, die vom Patienten gerade noch wahrgenommen werden können. Das Gerät wird an einem Kopfhörer für mehrere Stunden täglich zu Hause getragen. Die Behandlung erstreckt sich meist über mehrere Monate und kann danach auch bei Bedarf angewendet werden. Da sich der Tinnitus im Laufe der Behandlung verändert, muss mehrmals nachjustiert werden.

Vorerst wird die Therapie als Privatleistung angeboten (ca. 2500 Euro plus Arztkosten).

ST $=$

Weitere Infos sowie eine Liste der bereits geschulten Ärzte finden Sie unter www.anm-medical.com

\section{NACHTS REFLUXBESCHWERDEN}

\section{Schlafmittel sind keine gute Therapie}

Ein nächtlicher gastroösophagealer Säurereflux kann zu Schlafstörungen führen. Im Hinblick auf die Säure-Clearance der Speiseröhre kann dies allerdings günstig sein, da nächtliche Arousals Schluckmanöver induzieren. Jetzt konnte in einer Studie gezeigt werden, dass ein Schlafmittel, nämlich Zolpidem, refluxbedingte Arousals verhindert mit der Folge, dass die nächtliche ösophageale Säure-Expositionszeit zunimmt. Daraus ergibt sich, so Prof. Stephan Miehlke, Dresden, für den praktischen Alltag: Nächtliche Refluxbeschwerden sollten bei Patienten mit einer Refluxkrankheit keinesfalls mit Schlafmitteln bekämpft werden. STI -

18. Gastroenterologie-Update-Seminar am 5./6. März 2010 in Wiesbaden

\section{Der Natur abgeschaut}

\section{Essen wie ein Vögelchen}

Sich gesund zu ernähren, könne man von den Vögeln lernen, meint der Pharmakologe Navindra Seeram von der University of Rhode Island. Halt, halt! Nicht die Tierkadaver vom Straßenbelag kratzen. Die Fressgewohnheiten von Geiern hat Seeram nicht im Sinn. Sie müssen auch nicht Karl Valentins Ententraum verwirklichen und „einen Wurm, 25 cm gelb“ hinunterwürgen. Und Sie müssen auch nicht, wie es im Kinderlied heißt, in seltsamen Stellungen speisen: „Köpfchen unter Wasser..."

Der Pharmakologe hat Zugvögel beobachtet, die auf Block Island einen Zwischenstopp eingelegt und sich dort einen kleinen Snack gegönnt haben. Auch wenn es sich bei den Tieren eigentlich um Insekten- oder Körnerfresser handelte, bevorzugten sie auf Block Island stark gefärbte Beeren.

Durch die Anstrengung beim Vogelzug erleiden die Tiere einen oxidativen Stress, vermutet Seeram. Und die Antioxidanzien in den Beeren könnten diesem Stress entgegenwirken. Seerams Kollege Scott McWilliams will jetzt Vögel in den Windkanal stecken und anschließend mit Beeren füttern, um die These des Pharmakologen zu überprüfen.

Muss er nicht. Wir vertrauen Mutter Natur und unseren gefiederten Freunden auch so. Wir werden uns jetzt eine ordentliche Portion Tollkirschen holen. Die sind toll gefärbt und Vögel lieben das Zeug. Bestimmt wahnsinnig gesund.

REINHOLD MANHART 